\title{
Heat Transfer Investigation in Engine Exhaust-Type Pulsating Flow
}

\author{
Marco Simonetti $^{1}$, Christian Caillol ${ }^{1}$, Pascal Higelin ${ }^{1}$, Clément Dumand $^{2}$, Emmanuel Revol ${ }^{2}$ \\ ${ }^{1}$ PRISME Laboratory, University of Orleans \\ 6 Avenue du Parc Floral, Orleans, France \\ marco.simonetti@univ-orleans.fr \\ ${ }^{2}$ Groupe PSA \\ Route de Gizy, Vélizy-Villacoublay, France
}

\begin{abstract}
There are many engineering practical situations where heat is transferred under conditions of pulsating flow such as in the exhaust pipes of Internal Combustion Engines. In these conditions, heat transfer mechanism is affected by the pulsating flow parameters. The objective of the present work is to experimentally investigate heat transfers for pulsatile turbulent flows in a pipe. A unique experimental apparatus able to reproduce a pulsating flow representative of the engine exhaust has been designed. A stationary turbulent hot air flow with a Reynolds number of 30000, based on the time average velocity, is excited through a pulsating mechanism and exchanges thermal energy with a steel pipe. Pulsation frequency ranges from 10 to $95 \mathrm{~Hz}$. The effects of pulsation frequency and pipe length are evaluated. It has been observed that flow pulsation enhances convective heat transfers in comparison with the steady case. The test-bench architecture let us to evidence that, when the flow is excited with a pulsation frequency equal to one of the resonance modes of the system, a local maximum of the heat transfers rate appears. Such behaviour has been found to be independent of the pipe length. Results also show that the actual Nusselt correlations to predict convective heat transfer are inaccurate for pulsating flows, suggesting that new correlations which account pulsation effects have to be proposed.
\end{abstract}

Keywords: Convective Heat Transfer Enhancement, Pulsating Flow, Internal Combustion Engine, Waste Heat Recovery

\section{Introduction}

Pulsating or pulsatile flow is frequently encountered in engineering systems, motivating, in the past few years, specific studies on heat transfers in these conditions. However, some available results are contradictory and the main question is still open: Does pulsation enhance or else degrade heat transfer compared to steady flow. Pulsating flows have been investigated in many experimental configurations: Dec et al. [1], [2] have studied the impact of the pulsation frequency, pulsation amplitude, and the mean flow rate on heat transfers in the tail pipe of a pulse combustor. Spatially averaged Nusselt number computations have shown that heat transfers increase with both pulsation amplitude and frequency. Elshafei et al. [3] have studied heat transfer characteristics of pulsating turbulent air flow in a pipe heated at uniform heat flux over a range of $6.6<\mathrm{f}<68 \mathrm{~Hz}$ and $10^{4}<\mathrm{Re}<4 \times 10^{4}$. Turbulent bursting mode has been identified as an explanation of the observed heat transfer enhancement or decrease. Patel et al. [4] have experimentally studied the uniform wall heat flux condition case, in a more narrow range of the flow properties: $1<\mathrm{f}<3.3 \mathrm{~Hz}$ and $0.7 \times 10^{4}<\operatorname{Re}<1.65 \times 10^{4}$. They have also observed that the mean Nusselt number increases depending upon the frequency range. Similar studies [5]-[7] have been performed but this variety of experimental configurations has led to contradictory results, owing to the small number of the operating variables studied in a relatively narrow variation range. In the present study, an experimental apparatus has been designed to reproduce a pulsating hot air flow over a range of $10<\mathrm{f}<95 \mathrm{~Hz}$ and $10^{4}<\operatorname{Re}<5 \times 10^{4}$, such experimental conditions are representative of the engine exhaust; moreover, a specific method has been developed to measure instantaneous radial profiles of air temperature and velocity to evidence heat and momentum transport across one section of the pipe. The work presented in this paper only focuses on the time and space average characterization of the heat transfers using an analytical formulation, derived from the energy balance equation, to calculate the total time-average convective heat transfer for a pulsating turbulent flow. 


\section{Pulsating flow facility}

The developed test rig, schematically depicted in figure 1, can be decomposed in three macro different parts: the first one produces a hot stationary air flow, the second one transforms the stationary flow to a pulsating flow, and, in the last part in which the flow is develops, heat transfers are estimated.

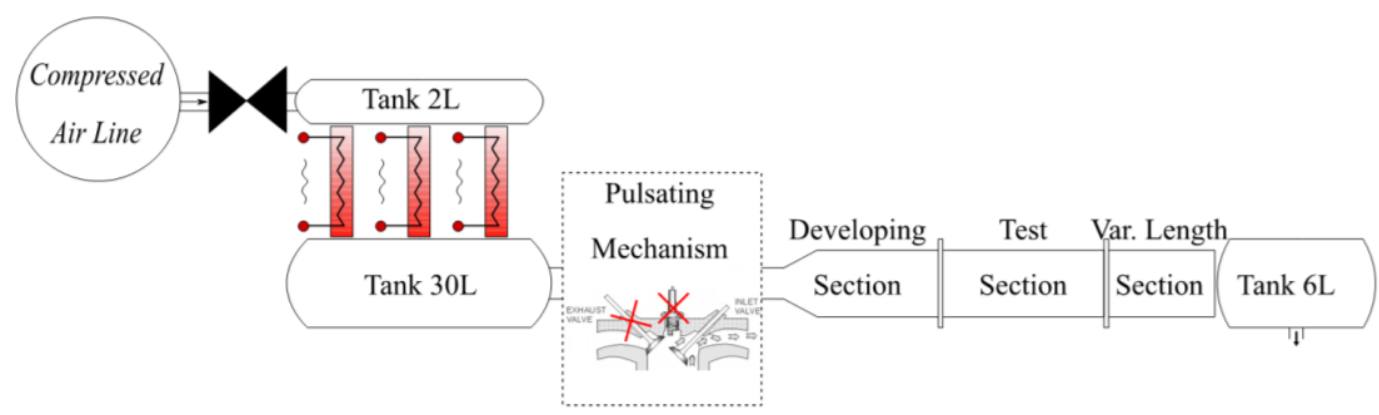

Fig. 1: Experimental apparatus.

In the first part of the test rig, the dry compressed air mass flow rate is measured and regulated by a Brooks SLA5853S, a thermal effect mass flow meter with a maximum flow rate of $2500 \mathrm{~nL} / \mathrm{min}$ and with a calibration incertitude of $0.73 \%$ of the full range scale. Then, air is heated by three 'Sylvania inline air heaters' with a total electric power of $12 \mathrm{~kW}$. 10 bars and to dumper the flow pulsation coming back from the pulse generator. Once the hot air flow has been generated it is forced to pass through the pulsating mechanism which consists of a mono-cylinder head equipped with a classical pushrod valve train entrained by an electric engine with a power of $3 \mathrm{~kW}$ and a nominal velocity of $3000 \mathrm{rpm}$ which corresponds to a maximum pulsation frequency of $95 \mathrm{~Hz}$. In details, hot air is flowed from the bottom of the cylinder head through one intake valve, which has been chosen because of its higher diameter. Air leakages have been experimentally estimated to be inferior to $0.5 \mathrm{~kg} / \mathrm{h}$ leading to an error on the mass flow rate measurement inferior to $1 \%$. In order to know the camshaft position and the rotation velocity an encoder with a resolution of $0.1^{\circ}$ is placed on the camshaft. Once the pulsating flow is generated, it is forced to pass through a steel pipe in which it develops and exchanges thermal energy. As shown in figure 1, the pipe is composed of three different sections: the first one, which has a length of 2.65 meters and a pipe length/diameter ratio of 48.4, is called developing section. It has been designed to be length enough to completely develop the cinematic field in the case of a steady turbulent flow. The pipe is thermal insulated to avoid high heat energy losses. The test section, with a length of 1 meter, is placed at the outlet of the developing section and is designed to be able to characterize the heat transfers. It consists in a double-wall pipe, in which the pulsating air and a cold water flow in opposite directions, respectively in the internal and external parts of the pipe. Water cooling has the advantages to be able to manage the wall temperature and to have a homogeneous temperature field in the internal wall of the pipe. A 'Kistler Type $2621 \mathrm{~F}$ conditioning unit', with a maximum cooling power up to 1500 Watt, is used to provide a maximum water flow rate of $6.1 \mathrm{~L} / \mathrm{min}$. The inlet and the outlet water temperatures are measured by $0.5 \mathrm{~mm}$ sheathed type $\mathrm{K}$ thermocouples. Same type thermocouples are placed in the test-section at five different sections spaced $100 \mathrm{~mm}$. In the first and last sections, four thermocouples are placed at different distances from the wall. In the remaining three sections, only the centreline temperature is measured. 'Kulite' pressure transducers are mounted at the inlet and outlet sections. As shown in figure 1, downstream the test-section a pipe with a variable length is used. The total length of the pipe can vary from 3.61 meters to 5.91 meters. Finally, at the end of the manifold a tank with a volume of 6 litres is placed to muffler pressure pulsation, which is finally linked to the exhaust line of the laboratory. A NI-9035 cRIO and the LabView software, are used to control all the devices in the experimental apparatus and to acquire data.

\section{Procedure \& Calculations}

The study of the impact of the flow pulsation on heat transfer phenomena is achieved by exciting a steady hot air flow with a pulsation frequency ranging from 0 to $95 \mathrm{~Hz}$. In practice, a mass flow rate, kept constant for all the experiments at $110 \mathrm{~kg} / \mathrm{h}$ is forced to flow through the mono-cylinder head. The centreline air temperature at the inlet of the test-section is maintained constant at $150^{\circ} \mathrm{C}$ for all experiments. The cooling water temperature at the inlet of the test-section is kept 
constant at $40^{\circ} \mathrm{C}$ for each experiment, with a maximum test-by-test variation of around $1{ }^{\circ} \mathrm{C}$. The pipe length variation generates a different acoustic response of the system subjected to a flow pulsation. Consequently, it is possible to change both the excitation frequency of the system and the system resonant frequency itself. The effect of the pulsation on heat transfers is determined in terms of the relative Nusselt number $N u_{r}$ defined as the ratio of the pulsating flow Nusselt number to the corresponding one for a steady flow at the same time-average Reynolds number. The Nusselt number is defined as in the following equation:

$$
N u=\frac{h D}{\lambda}=\frac{Q_{c o n v} D}{S \Delta T_{l m} \lambda}
$$

Where: $Q_{c o n v}$ is the convective heat transfer, $D$ the internal diameter of the pipe equal to $54.7 \mathrm{~mm}, S$ the exchange surface, $\Delta T_{l m}$ the logarithmic mean temperature difference between the air and the internal wall of the pipe and $\lambda$ the thermal conductivity of air. To be coherent with literature correlations for the Nusselt number, the air temperature at the inlet and the outlet of the test-section corresponds to the bulk temperature, computed from the four thermocouples placed in these sections. The total mean convective heat transfer $Q_{c o n v}$ is calculated applying the energy balance equation to air. Starting from the 1D energy balance equation for a turbulent pulsating flow in which the triple-decomposition, proposed by Reynolds et al. [8], is introduced, then applying phase $(<>)$ and time $\left(^{-}\right)$averaging operators, and finally integrating the local equation on the control volume (between the inlet and outlet measuring sections of the test section), the total mean convective heat transfer has been identified as in equation 2. Fluid incompressibility, negligible viscous dissipation and heat conduction have been assumed.

$$
\rho c_{p} S(\underbrace{\left.\bar{u} \bar{T}\right|_{0} ^{L}}_{A}+\underbrace{\overline{\left.\tilde{u} \tilde{T}\right|_{0} ^{L}}}_{B}+\underbrace{\overline{\left\langle u^{\prime} T^{\prime}\right.}>\left.\right|_{0} ^{L}}_{C})=\int_{V} \overline{\langle q(x, t)\rangle} d V=Q_{c o n v}
$$

In the above equation $u$ represents the bulk axial instantaneous air velocity, T the bulk temperature of air, $\rho$ the fluid density, $c_{p}$ the specific heat at constant pressure, $q$ the local specific convective heat transfer exchanged through the perimeter of a pipe length $\partial x$.

Such analytical approach highlights that the total time-average convective heat transfer $Q_{c o n v}$ is composed by the sum of three different terms: 'A' which physically represents the energy variation of the time-mean component of the flow across a pipe length $\mathrm{L}$, ' $\mathrm{B}$ ' which represents the energy variation due to the oscillating component of the flow and ' $\mathrm{C}$ ' which represents the energy which propagates by the advection due to turbulence. It has been observed that the term ' $\mathrm{B}$ ' strongly depends on the boundary conditions of the test section: in the case of flow reversal combined with a pipe temperature, downstream the test-section, greater that the flow temperature, energy can be introduced through the control surfaces of the test-section leading to a negative value of the term ' $\mathrm{B}$ ', which is not physical. Moreover, assuming that the variation of the pulsation frequency does not significantly impact the turbulence properties, it can be considered that the term ' $C$ ' does not modify the convective heat transfer when the pulsation frequency is changed. Consequently, only the term 'A' of Eq. 2 has been considered to calculate $Q_{c o n v}$ from the time-average value of air temperatures at the inlet and outlet sections of the test-section. All the fluid properties are calculated at the mean bulk temperature. Uncertainty analysis of the Nusselt number has been carried out by calculating the maximum and minimum Nusselt number values, accounting for the standard deviation of the temperature signals. Uncertainties are depicted by error bars in the following figures.

\section{Results \& Discussion}

Figure 2 shows the relative Nusselt number with the relative incertitude in function of the flow pulsation frequency, for the case with the maximum and minimum length of the pipe (L3 and L1 respectively). It can be evidenced that for all the applied frequencies range heat transfers are improved in comparison to the steady case. Thanks to a previous characterization of the acoustic resonance modes of the pipe, it has been possible to correlate the local maxima of the relative Nusselt number to the system resonance. For instance, for the L3 particular case, system resonance is in correspondence to the $2^{\text {nd }}, 3^{\text {rd }}$ and $4^{\text {th }}$ resonance modes, respectively $22.2,49.5$ and $80.5 \mathrm{~Hz}$. When the experimental apparatus is subjected to a pulsating flow, in which the pulsating frequency is equal to one of the resonance modes of the 
pipe, an enhancement of the convective heat transfer component, due to the energy advected through the time mean component of the flow, occurs. It can be supposed that a resonance of the system may lead to an enhanced oscillating component of the velocity, but direct experimental evidences are not still available. To have further useful information on the instantaneous heat and momentum transport across the boundary layer in a pipe section, in order to understand the mechanisms of the heat transfer enhancement, instantaneous measurements of the flow velocity, temperature radial profiles and static pressure will be conducted. A double parallel wires anemometer and micro unsheathed thermocouples will be used to measure both air temperature, and the magnitude and direction of the axial component of the air velocity.

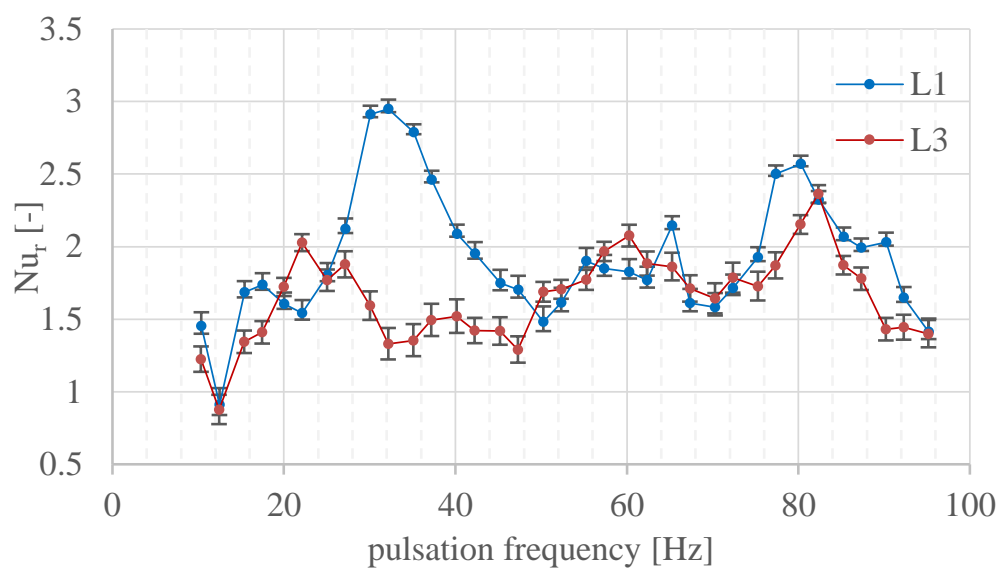

Fig. 2: Impact of the Pipe's Length on Heat Transfers.

\section{Conclusion}

In this study an investigation on the heat transfers for a pulsating flow has been conducted. A specific experimental apparatus has been designed to reproduce a pulsating turbulent flow representative of the engine exhaust. Pulsation frequency has been varied from $10 \mathrm{~Hz}$ to $95 \mathrm{~Hz}$ for a time-average Reynolds number of 30000. A particular approach to evidence coherent phenomena in the heat transfer enhancement has been adopted: the length of the pipe in which the flow develops and exchanges thermal energy has been systematically varied. Results show that in the case of a resonant flow, in which the pulsation source excites the flow with a frequency equal to one of the resonant modes of the system, a heat transfer enhancement appears. Such behaviour has been found to be independent of the pipe length, confirming that a coherent mechanism in the heat transfer enhancement exists. Although this first macroscopic study doesn't allow explaining in detail the heat transfer mechanisms, it has provided important results for a further investigation in which instantaneous measurements of the flow properties across a section of the pipe will be conducted to characterize the heat and momentum transport in the boundary layer.

\section{Acknowledgements}

This work was supported by the 'OpenLab Energetics' program, thanks to the partnership between the Groupe PSA and the PRISME Laboratory of the University of Orleans.

\section{References}

[1] J. E. Dec and J. O. Keller, "Pulse combustor tail-pipe heat-transfer dependence on frequency, amplitude, and mean flow rate," Combust. Flame, vol. 77, no. 3-4, pp. 359-374, 1989.

[2] J. E. Dec, J. O. Keller, and V. S. Arpaci, "Heat transfer enhancement in the oscillating turbulent flow of a pulse combustor tail pipe," Int. J. Heat Mass Transf., vol. 35, no. 9, pp. 2311-2325, 1992.

[3] E. A. M. Elshafei, M. Safwat Mohamed, H. Mansour, and M. Sakr, "Experimental study of heat transfer in pulsating turbulent flow in a pipe," Int. J. Heat Fluid Flow, vol. 29, no. 4, pp. 1029-1038, 2008.

[4] J. T. Patel and M. H. Attal, "An Experimental Investigation of Heat Transfer Characteristics of Pulsating Flow in Pipe," Int. J. Curr. Eng. Technol., vol. 6, no. 5, pp. 1515-1521, 2016.

[5] P. K. Papadopoulos and A. P. Vouros, "Pulsating turbulent pipe flow in the current dominated regime at high and very-high frequencies," Int. J. Heat Fluid Flow, vol. 58, pp. 54-67, 2016. 
[6] E. M. Benavides, "Heat transfer enhancement by using pulsating flows," J. Appl. Phys., vol. 105, no. 9, p. 94907, 2009.

[7] M. a. Habib, S. a. M. Said, a. a. Al-Farayedhi, S. a. Al-Dini, a. Asghar, and S. a. Gbadebo, "Heat transfer characteristics of pulsated turbulent pipe flow," Heat Mass Transf., vol. 34, no. 5, pp. 413-421, 1999.

[8] W. C. Reynolds and a. K. M. F. Hussain, "The mechanics of an organized wave in turbulent shear flow. Part 3. Theoretical models and comparisons with experiments," J. Fluid Mech., vol. 54, p. 263, 1972. 